\title{
PROMOCIÓN DE LA DEMANDA DE INNOVACIÓN TECNOLÓGICA
}

Héctor W. Cardoso $\left(^{*}\right)$

Palabras claves: promoción de la innovación productiva, promoción de la innovación tecnoló-gica, el promotor de innovación tecnológica, I.T., I.P., SINTEC.

Se describe un modelo de gestión que se realiza en el SINTEC cuya metódica persigue la inno-vación tecnológica (I.T.) a partir de la demanda. Sus modalidades de trabajo surgieron de una experiencia que comenzara en el año 2001 y continúa hasta la fecha.

\section{Objetivos}

- Promover la I.T. local a partir de la demanda, detectando las necesidades que existen al res-pecto, realizando una gestión capaz de inducir en los sistemas tecnocientíficos el desarrollo de ofertas en tal sentido e impulsando la apropiación social de la tecnología.

- Promover un diálogo fructífero y respetuoso entre todos los saberes, el pensamiento crítico e integrador para interpretar una realidad humana compleja y fragmentaria ante la futilidad y deterioro de los compartimentos estancos del régimen disciplinar que prima en los sistemas tradicionales.
- Desarrollar un espacio formativo y educativo que interrelacione diversas disciplinas, un es-pacio comprometido con la promoción humana, el acceso y el intercambio de conocimientos, el desarrollo de la identidad cultural de los pueblos, que tenga como valores: democracia, desarrollo sustentable, participación, justicia, equidad, solidaridad, derechos humanos, plura-lismo, cooperación.

- Formar y educar nuevos profesionales mediante una metodología que estimule iniciativas a partir de tareas prácticas.

\section{Métodos}

Todo lo realizado en el SINTEC se orientó siempre en la búsqueda de operacionalizar la idea de generar innovación tecnológica a partir de la demanda. En la actualidad la experiencia acumulada ha permitido sistematizar en buena medida la metódica del trabajo del Promotor de Innovación Tecnológica (PIT).

El SINTEC es un sistema que opera llevando innovación productiva y tecnológica para el desa-rrollo local, actúa en los municipios en coordinación con la autoridad municipal.

(*) Director del Sistema Provincial de Innovación Tecnológica. (SINTEC) dependiente del Comité Ejecutivo de Desarrollo e Innovación Tecnológica de Misiones (CEDIT). Se trata de un orga-nismo perteneciente al Ministerio de Cultura, Educación, Ciencia y Tecnología de la provincia de Misiones. Dirección: Félix de Azara 1890 - 5to. Piso, (N3300LQN) Posadas, Misiones - Teléfonos: +54 (3764) 447005 / 447019. Cel.: +54 (3764)240441 - hectorwcardoso@yahoo.com.ar 
Se vale de los PIT que básicamente deben realizar diagnósticos de necesidades de I.T., efectuar gestiones de vinculación y formular y participar en proyectos de transferencia.

En la actualidad la experiencia ganada con el trabajo de campo ha permitido definir un verdadero oficio que requiere de considerable compromiso, iniciativa y creatividad para que una tecnología sea desarrollada, adaptada, aceptada, se concrete y prospere en el tiempo. Se trata de un trabajo multidisciplinario y de gestión intervinculante, en el que se debe tener en cuenta innumerables aspectos que hacen tanto a la valoración intrínseca de la tecnología como a sus condicionantes económicos, sociales y ambientales.

En este proceso de diagnóstico, vinculación y transferencia se intenta la participación de la po-blación local en la definición de sus necesidades de I.T. considerando la posibilidad real de poder apropiarse de ella y de lograr un desarrollo en lo social y económico asegurando la sustentabilidad ambiental. En base a todo esto se trata de elaborar la demanda de innovación productiva y/o tecnológica que luego en el proceso de vinculación influya en la generación de la oferta por parte de los organismos de ciencia y tecnología.

Consideramos que el desarrollo de una comunidad está condicionado, entre otras cosas, por la posesión que ésta tenga de tecnologías capaces de resolver sus necesidades. Por esta razón son los mismos actores locales, que experimentan esas necesidades, quienes están en mejores condiciones para iniciar procesos de desarrollo genuino.

En su proceder el SINTEC busca generar tecnología operando desde lo social. Se trata de lograr la participación activa de personas e instituciones que se identifican como pertenecientes a un determinado lugar (población rural, pueblo, barrio, ciudad, etc.).

Hay que reconocer que el proceso de transferencia de I.T. no siempre constituye una tarea simple, esto es debido a que debe darse en un marco de condicionamientos sociales, económicos, ambientales y que, muchas veces, plantea la necesidad del cambio cultural.

Toda vez que la gestión de la innovación parte de las necesidades que tiene una población, surgen dificultades para el cambio innovador si tenemos en cuenta que esas necesidades comúnmente no son percibidas ni sentidas, la demanda expresada se presenta en el menor de los casos, especialmente en comunidades rurales con componentes tradicionales arraigados.

Los PIT diferencian en su accionar la innovación productiva (I.P.) de la tecnológica.

Innovación Productiva: forma productiva localmente nueva, que incorpora tecnología -no nece-sariamente innovadora- y que requiere por lo general de un proceso previo de inducción y aseso-ramiento para su aceptación, apropiación y manejo por parte de los productores.

Innovación Tecnológica: sistema tecnológico nuevo que permite transformar y/o controlar nuevos sectores de la realidad, que mejora las propiedades de uno preexistente (especialmente en eficiencia) o que lo modifica para hacerlo más adecuado a las condiciones locales.

Se citan las principales actividades que realizan los PIT: detectar necesidades y demandas de IP e IT, buscar conocimientos, participar en los ateneos, acompañar a pro- 
cesos de innovación, realizar coordinaciones intersectoriales, divulgar, capacitar, asesorar, gestionar procesos de vinculación, detectar desarrollos tecnológicos necesarios, detectar investigaciones necesarias, formular de proyectos, realizar transferencias, gestionar la formalización de emprendimientos, evaluar impactos. Estas actividades se describen en material didáctico.

El SINTEC es una red que se constituye con nodos, uno por municipio, en los que se desempeñan los PIT.Tiene una Unidad Central de administración. Cuando los nodos detectan necesidades de IT se inician las tareas de transferencia cuando se requiere de una innovación productiva, si es posible mediante la presentación de la correspondiente oferta ya conocida o disponible en el lugar, cuando se requiere de una I.T. se realizan consultas dentro de la red o se derivan las de-mandas directamente a diversas instituciones de investigación y desarrollo o a la citada unidad central. Aquí se da curso a las diferentes demandas, son derivadas a instituciones dedicadas al tema.

Si no hay posibilidades de generar las correspondientes ofertas en el medio provincial se deriva el problema a las instituciones extra provinciales. Una vez en posesión de la oferta se inicia el trabajo de transferencia.

La unidad central además concentra la información necesaria para toda la provincia y la distribu-ye de acuerdo a las necesidades que surgen en los municipios.

\section{Ateneo de C\&T}

Es una reunión de todos los promotores que se hace una vez por mes.

En el ateneo participan profesionales (PIT) de diferentes disciplinas que dialogan entre sí a partir de las exposiciones que cada uno hace respecto de las actividades que está realizando, manifies-tan sus dudas y detallan sus propuestas.

La experiencia acumulada nos dice que no habrá innovación si en la reunión se expone exclusi-vamente acerca de "lo que se hace" habitualmente en materia tecnológica. La originalidad surge del cuestionamiento, de la crítica, especialmente dirigida al "porqué se hace" lo observado y lue-go, a partir de allí, proponer "el hacer" para lograr el cambio tecnológico en cada caso. Cobra importancia plantear claramente el problema detectado en torno de alguna necesidad que pueda ser resuelta con una innovación.

El grupo revisa y analiza lo realizado, se debaten los diferentes problemas y alternativas para solucionarlos. $\mathrm{El}$ ateneo es un ámbito multidisciplinar, en el se establece un debate entre los dife-rentes saberes disciplinares. Los diversos enfoques, ora críticos ora constructivos, parten de dis-tintas concepciones.

Se llega a resultados que enriquecen las experiencias individuales por el aporte de expertos en diferentes materias. Las diferentes intervenciones permiten descentrar las miradas construidas sobre la base de una disciplina única. A través del debate y el intercambio multidisciplinar se construyen ideas y concepciones novedosas.

En el ateneo se cumple un proceso creativo en el que la conversación adquiere una singular im-portancia.

En la "presentación de casos" los tres temas principales son: a) Dificultades en la realización de experiencias de diagnóstico, vinculación o transferencia. b) Problemas que requieren de la for-mulación de un proyecto. Para la presentación del caso el PIT describe: situación problema, objetivos que se evalúan, antecedentes, ideas para un 
proyecto. c) Dificultades que surgen en proyecto en curso.

\section{Proceso de aprendizaje del PIT}

La pasantía en la promoción de la I.T. de la Provincia de Misiones está destinada al graduado reciente, se trata de un ejercicio de capacitación orientado a la detección de necesidades existen-tes en la sociedad que se resuelven con la innovación Esta pasantía constituye una modalidad de aprendizaje por autogestión orientada.

Debido a que en este sistema es central la promoción del trabajo innovador, aquí es el propio pasante el que debe detectar las necesidades sociales de nuevos conocimientos, buscar los ya logrados en otros ámbitos y generar finalmente la innovación.

Se trata de promover las iniciativas y la creatividad en el joven profesional. El apoyo externo que brindan instructores y supervisores permite orientar los procesos de aprendizaje y estímulo al trabajo innovador. En un comienzo con mayor participación del instructor, luego gradualmente con mayor participación del pasante y menor del instructor hasta que el pasante comienza con un aprendizaje autodidacta.

Simultáneamente este sistema constituye un entrenamiento preparatorio para una futura actividad laboral, de esta manera se supera más fácilmente el bache existente entre la universidad y la realidad productiva.

En ambos sentidos esta pasantía es complementaria con el sistema universitario tradicional.

Los promotores reciben inicialmente material didáctico que instruye acerca del trabajo que deben realizar, luego todo lo que hagan será iniciativa de cada uno incluyendo nuevos aportes que pue-dan enriquecer al oficio que desempeñan.

Esta pasantía tiene una duración máxima de dos años, con renovación, luego del primer año por evaluación.

\section{Conclusiones}

Este modelo de gestión ha dado excelentes resultados en materia de innovación productiva, los PIT han resuelto dos carencias de importancia en los municipios: la falta de llegada a los sectores más necesitados y la falta de formuladores de proyectos. No puede decirse lo mismo de la inno-vación tecnológica, hay promotores que tienen dificultad para lograr la detección de una necesi-dad que implique innovación, lo más fácil es percatarse de las insuficiencias de una tecnología vigente y buscar sus mejoras o su reemplazo. Generalmente la elaboración de una demanda con-creta surge en el ateneo. Otra limitación es que las interacciones de vinculación no siempre encuentran eco en los sistemas tecno-científicos tradicionales.

De todos modos se trata de una iniciativa de gestión, que teniendo continuidad y desarrollo, se presenta válida para realizar aportes a la necesidad de construir un conocimiento capaz de integrar los diversos dominios del saber, desde las especialidades del claustro universitario hasta el saber popular de las culturas nativas. Para lograr un conocimiento pertinente e integrador desde lo social capaz de encarar adecuadamente los cambios que se imponen en tiempos de incertidumbre. 

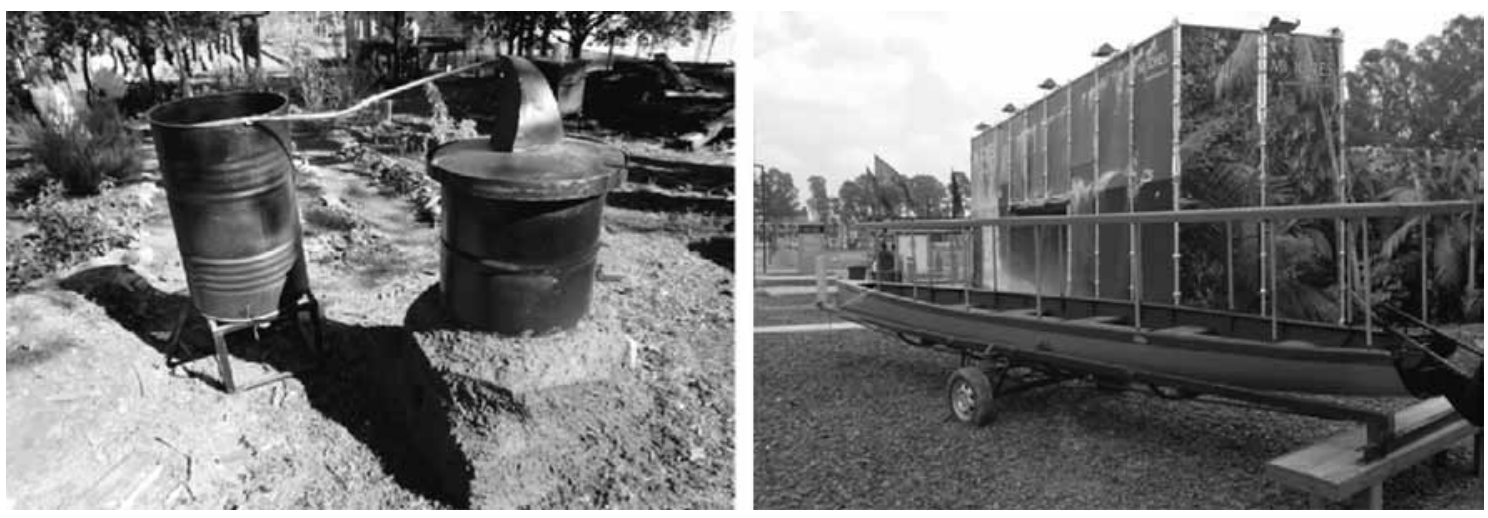

Dos casos extremos en el nivel de desarrollo de ofertas de I.T. basadas en demandas registradas por PITs.

Izquierda alambique en escuela rural del municipio de Fachinal. Tecnología apropiada realizable por autoconstrucción. Se utilizaron tambores de desecho y horno de barro tradicional. Desarrollada para ahorrar leña a pedido de pequeños productores que elaboran aceites esenciales. (Innovación tecnológica más no productiva por demanda orientada).

Derecha canoa a energía solar exhibida en Tecnópolis. Desarrollada ante la necesidad de emprendedores en ecoturis-mo que operan en el Corredor Iberá-Yabotí de contar con medios de bajo impacto para acceder al ecosistema. La oferta, más allá del diseño tecnológico, fue concebida a partir de una necesidad percibida pero no especificada por los emprendedores. (Innovación productiva y tecnológica por demanda no orientada).

\section{Bibliografía}

- Documentos del MINCYT (Ministerio de Ciencias, Tecnología e Innovación Productiva de la Argentina).

- Documentos diversos del CIET (Centro Internacional de Estudios Transdisciplinarios). Ciudad de México.

- Héctor Cardoso, Marcelo Orué, Silvia Pona, Marcelo Sarasola, Eduardo Puppio. EL PROMOTOR DE INNOVACIÓN TECNOLÓGICA. Su capacitación. Graffi. Posadas Misiones 2004.

- Vandana Shiva, Carlo Petrini, Fitjof Capra, José Esquinas-Alcázar, Marcello Buiatti, Benny Haerlin, Gianluca Brunori, Caroline Lockhart y otros. MANIFIESTO SOBRE EL FUTURO DE LOS SISTEMAS DE CONOCIMIENTO. Soberanía del Conocimiento para un Planeta Sano. Arsia. 2009. 